Proceedings of the Edinburgh Mathematical Society (2006) 49, 551-565 (C)

DOI:10.1017/S0013091505000301 Printed in the United Kingdom

\title{
THE EXISTENCE OF ISOTROPIC MODULI SPACES
}

\author{
MD. SHOWKAT ALI \\ Department of Mathematics, University of Dhaka, Dhaka 1000, Bangladesh \\ (msa417@hotmail.com)
}

(Received 9 March 2005)

\begin{abstract}
A theorem on the existence of moduli spaces of compact complex isotropic submanifolds in complex contact manifolds is established.

Keywords: contact manifold; isotropic; Legendre and Kodaira moduli spaces

2000 Mathematics subject classification: Primary 53D10; 53D30; 32G10
\end{abstract}

\section{Introduction}

In 1962 Kodaira [3] proved that if $X \hookrightarrow Y$ is a compact complex submanifold of a complex manifold $Y$ with normal bundle $N_{X \mid Y}$ such that $H^{1}\left(X, N_{X \mid Y}\right)=0$, then there exists a complete analytic family $\left\{X_{t} \hookrightarrow Y \mid t \in M\right\}$ of compact complex submanifolds $X_{t}$ of $Y$ with the moduli space $M$. The family is maximal and its moduli space $M$, called the Kodaira moduli space, is an $h^{0}\left(X, N_{X \mid Y}\right)$-dimensional complex manifold. Kodaira's theorem found many applications in geometry and analysis, especially in twistor theory. Merkulov [7] proved that if $X \hookrightarrow Y$ is a compact complex Legendre submanifold of a complex contact manifold $Y$ with contact line bundle $L$ such that $H^{1}\left(X, L_{X}\right)=0$, then there exists a complete and maximal analytic family $\left\{X_{t} \hookrightarrow Y \mid t \in M\right\}$ of compact Legendre submanifolds containing $X$ with the moduli space $M$, which is an $h^{0}\left(X, L_{X}\right)$-dimensional complex manifold. In this paper we prove that if $X \hookrightarrow Y$ is a compact complex isotropic submanifold of a complex contact manifold $Y$ with contact line bundle $L$ such that $H^{1}\left(X, L_{X}\right)=H^{1}\left(X, S_{X}\right)=0$, where $L_{X}$ is the restriction of $L$ on $Y$ to $X$ and $S_{X}$ is a certain canonically defined vector bundle on $X$ which is the kernel of the canonical projection $p: N_{X \mid Y} \rightarrow J^{1} L_{X}$, then there exists a complete and maximal analytic family $\left\{X_{t} \hookrightarrow Y \mid t \in M\right\}$ of compact isotropic submanifolds containing $X$ with the moduli space $M$, which is an $h^{0}\left(X, L_{X}\right)+h^{0}\left(X, S_{X}\right)$-dimensional smooth complex manifold. There are strong indications in $[8]$ that the moduli spaces of such families studied in this paper will play a pivotal role in the twistor theory of $G$-structures with restricted invariant torsion. 


\section{Complex contact manifolds}

Definition 2.1. A complex contact manifold is a pair $(Y, D)$ consisting of a $(2 n+1)$ dimensional complex manifold $Y$ and a rank- $2 n$ holomorphic subbundle $D \subset \mathcal{T} Y$ of the holomorphic tangent bundle to $Y$ such that the Frobenius form

$$
\begin{gathered}
\phi: D \times D \rightarrow \mathcal{T} Y / D, \\
(v, w) \rightarrow[v, w] \bmod D
\end{gathered}
$$

is non-degenerate. Define the contact line bundle $L:=\mathcal{T} Y / D$ on $Y$ by the exact sequence

$$
0 \rightarrow D^{2 n} \rightarrow \mathcal{T} Y^{2 n+1} \stackrel{\theta}{\rightarrow} L \rightarrow 0,
$$

where $\theta$ is the tautological projection and $D=\operatorname{ker} \theta$. However, we may also think of $\theta$ (in a trivialization of $L)$ as a line bundle-valued 1-form $\theta \in H^{0}\left(Y, \Omega^{1} Y \otimes L\right)$, and so attempt to form its exterior derivative $\mathrm{d} \theta$. We can easily verify that the maximal non-degeneracy of the distribution $D$ is equivalent to the fact that the 'twisted' 1 -form defined above satisfies the condition

$$
\theta \wedge(\mathrm{d} \theta)^{n} \neq 0
$$

\section{Complex isotropic submanifolds}

Definition 3.1. A compact complex $p$-dimensional submanifold $X^{p} \hookrightarrow Y^{2 n+1}$ of a complex contact manifold $Y^{2 n+1}$ is called isotropic if $\left.\mathcal{T} X \subset D\right|_{X}$.

An isotropic submanifold of maximal possible dimension $n$ is called a Legendre submanifold. The normal bundle $N_{X \mid Y}$ of any Legendre submanifold $X \hookrightarrow Y$ is isomorphic to $J^{1} L_{X}[\mathbf{5}]$, where $L_{X}=\left.L\right|_{X}$, and, therefore, fits into the exact sequence

$$
0 \rightarrow \Omega^{1} X \otimes L_{X} \rightarrow N_{X \mid Y} \stackrel{\mathrm{pr}}{\longrightarrow} L_{X} \rightarrow 0 .
$$

Definition 3.2. The bundle $S_{X}$ is defined to be the kernel of the canonical projection

$$
p: N_{X \mid Y} \rightarrow J^{1} L_{X}
$$

i.e. it is defined by the exact sequence

$$
0 \rightarrow S_{X} \rightarrow N_{X \mid Y} \rightarrow J^{1} L_{X} \rightarrow 0 .
$$

Definition 3.3. Let $X$ be an isotropic submanifold of a complex contact manifold $(Y, D)$. Let

$$
T X^{\perp}=\{Z \in D \mid \mathrm{d} \theta(Z, W)=0, \forall W \in T X\} .
$$

Then $T X \subseteq T X^{\perp}$ and the bundle $S_{X}$ is defined by $S_{X}=T X^{\perp} / T X$.

Theorem 3.4. Let $(Y, D)$ be a complex contact manifold and $X \subset Y$ be an isotropic submanifold of $Y$ with contact line bundle $L$. Then there is a short exact sequence

$$
0 \rightarrow S_{X} \rightarrow N_{X \mid Y} \rightarrow J^{1} L_{X} \rightarrow 0
$$


Proof. Consider a particular 1-form $\theta$ that represents the contact structure. Let $p \in$ $X, Z \in T_{p} X$ be a vector in the normal bundle and $Q \in T_{p} Y$. There are then two equations

$$
f(p)=\theta(Q), \quad \mathrm{d} \theta(Z, Q)=\left.Z(f)\right|_{p},
$$

which uniquely determine the 1-jet on $X$ of a function $f$ at $p$.

Consider rescaling $\theta \mapsto g \theta$, where $g$ is a function on $Y$. If we set $\hat{\theta}=g \theta$ and $\hat{f}=g f$, then we have

$$
\hat{\theta}(Q)=g \theta(Q)=g f(p)=\left.\hat{f}\right|_{p}
$$

and

$$
\begin{aligned}
\mathrm{d} \hat{\theta}(Z, Q) & =(\mathrm{d} g \wedge \theta)(Z, Q)+g \mathrm{~d} \theta(Z, Q) \\
& =\mathrm{d} g(Z) \theta(Q)-\mathrm{d} g(Q) \theta(Z)+\left.g Z(f)\right|_{p} \\
& =Z(g) f(p)-0+\left.g Z(f)\right|_{p} \\
& =\left.Z(g f)\right|_{p} \\
& =\left.Z(\hat{f})\right|_{p} .
\end{aligned}
$$

(Since $T_{p} X \subseteq T_{p} X^{\perp} \subset D$, we have $Z \in D$, so $\theta(Z)=0$.) Therefore, this elementary calculation shows that the two conditions above are satisfied by $g f$ and so we can conclude that we have defined a map $N_{X \mid Y} \rightarrow J^{1} L_{X}$. Furthermore, it is clear that the kernel is $T X^{\perp} / T X$. Thus, the proof is completed.

\section{Kodaira relative deformation theory}

In this section we recall some useful facts about relative deformation theory of compact complex submanifolds of complex manifolds $[\mathbf{6}]$.

Let $Y$ and $M$ be complex manifolds and let $\pi_{1}: Y \times M \rightarrow Y$ and $\pi_{2}: Y \times M \rightarrow M$ be two natural projections. An analytic family of compact submanifolds of the complex manifold $Y$ with moduli space $M$ is a complex submanifold $F \hookrightarrow Y \times M$ such that the restriction of the projection $\pi_{2}$ on $F$ is a proper regular map (regularity means that the rank of the differential of $\left.\nu \equiv \pi_{2}\right|_{F}: F \rightarrow M$ is equal to $\operatorname{dim} M$ at every point). Thus, the family $F$ has double fibration structure

$$
Y \stackrel{\mu}{\longleftarrow} F \stackrel{\mu}{\rightarrow} M
$$

where $\mu=\left.\pi_{1}\right|_{F}$. For each $t \in M$ we say that the compact complex submanifolds $X_{t}:=$ $\mu \circ \nu^{-1}(t) \hookrightarrow Y$ belong to the family $F$.

\section{Existence of Legendre moduli spaces}

Let $Y$ be a complex contact manifold. An analytic family $F \hookrightarrow Y \times M$ of compact submanifolds of $Y$ is called an analytic family of compact Legendre submanifolds if, for any point $t \in M$, the corresponding subset $X_{t}:=\mu \circ \nu^{-1}(t) \hookrightarrow Y$ is a Legendre submanifold. The parameter space $M$ is called a Legendre moduli space. In 1995, Merkulov [7] proved the following theorem for the existence of complete Legendre moduli spaces. 
Theorem 5.1 (Merkulov [7]). Let $X$ be a compact complex Legendre submanifold of a complex contact manifold $Y$ with contact line bundle $L$. If $H^{1}\left(X, L_{X}\right)=0$, then there exists a complete and maximal analytic family $\left\{X_{t} \hookrightarrow Y \mid t \in M\right\}$ of compact Legendre submanifolds containing $X$ with the moduli space $M$, which is an $h^{0}\left(X, L_{X}\right)$-dimensional complex manifold.

\section{Families of complex isotropic submanifolds}

Let $Y$ be a complex contact manifold. An analytic family $F \hookrightarrow Y \times M$ of compact submanifolds of the complex manifold $Y$ is called an analytic family of isotropic submanifolds if, for any $t \in M$, the corresponding subset $X_{t}=\mu \circ \nu^{-1}(t) \hookrightarrow Y$ is an isotropic submanifold. We will use the notation $\left\{X_{t} \hookrightarrow Y \mid t \in M\right\}$ to denote an analytic family of isotropic submanifolds.

Let $X=X_{t_{0}}$ for some $t_{0} \in M$. If $X^{p} \hookrightarrow Y^{2 n+1}$ is an isotropic submanifold, then each point in $X$ has a neighbourhood $U$ in $Y$ such that the contact structure in a suitable trivialization of $L$ over $U$ (see [2]) is

$$
\theta=\mathrm{d} \omega^{0}+\sum_{\bar{a}=p+1}^{n} \omega^{\bar{a}} \mathrm{~d} \omega^{\overline{\bar{a}}}+\sum_{a=1}^{p} \omega^{a} \mathrm{~d} z^{a}
$$

and $X$ in $U$ is given by

$$
\omega^{0}=\omega^{a}=\omega^{\bar{a}}=\omega^{\overline{\bar{a}}}=0 .
$$

There exists an adopted coordinate covering $\left\{U_{i}\right\}$ of a tubular neighbourhood of $X$ inside $Y$. In view of the above fact one can always choose local coordinate functions $\left(\omega_{i}^{0}, \omega_{i}^{a}, \omega_{i}^{\bar{a}}, \omega_{i}^{\bar{a}}, z_{i}^{a}\right)$ in $U_{i}$, where $\bar{a}, \overline{\bar{a}}=1, \ldots, n$ and $a=1, \ldots, p$ such that the contact structure in $U_{i}$ is represented by

$$
\theta_{i}=\mathrm{d} \omega_{i}^{0}+\sum_{\bar{a}=p+1}^{n} \underbrace{\omega_{i}^{\bar{a}} \mathrm{~d} \omega_{i}^{\overline{\bar{a}}}}_{(n-p) \text {-terms }}+\sum_{a=1}^{p} \underbrace{\omega_{i}^{a} \mathrm{~d} z_{i}^{a}}_{p \text {-terms }}
$$

and $U_{i} \cap X$ is given by

$$
\omega_{i}^{0}=\omega_{i}^{a}=\omega_{i}^{\bar{a}}=\omega_{i}^{\overline{\bar{a}}}=0
$$

and

$$
\left.\theta_{i}\right|_{U_{i} \cap U_{j}}=\left.A_{i j} \theta_{j}\right|_{U_{i} \cap U_{j}}
$$

for some nowhere-vanishing holomorphic functions $A_{i j}$. They satisfy the condition

$$
A_{i k}=A_{i j} A_{j k}
$$

on every triple intersection $U_{i} \cap U_{j} \cap U_{k}$. Clearly, $\left\{A_{i j}\right\}$ are glueing functions of the contact line bundle $L$. 
On the intersection $U_{i} \cap U_{j}$, the coordinates $\omega_{i}^{A}:=\left(\omega_{i}^{0}, \omega_{i}^{a}, \omega_{i}^{\bar{a}}, \omega_{i}^{\overline{\bar{a}}}\right)$ and $z_{i}^{a}$ are holomorphic functions of $\omega_{j}^{B}:=\left(\omega_{j}^{0}, \omega_{j}^{a}, \omega_{j}^{\bar{a}}, \omega_{j}^{\bar{a}}\right)$ and $z_{j}^{b}$,

$$
\left.\left.\begin{array}{rl}
\omega_{i}^{0} & =f_{i j}^{0}\left(\omega_{j}^{B}, z_{j}^{b}\right) \\
\omega_{i}^{a} & =f_{i j}^{a}\left(\omega_{j}^{B}, z_{j}^{b}\right) \\
\omega_{i}^{\bar{a}} & =f_{i j}^{\bar{a}}\left(\omega_{j}^{B}, z_{j}^{b}\right) \\
\omega_{i}^{\bar{a}} & =f_{i j}^{\overline{\bar{a}}}\left(\omega_{j}^{B}, z_{j}^{b}\right) \\
z_{i}^{a} & =g_{i j}^{a}\left(\omega_{j}^{B}, z_{j}^{b}\right)
\end{array}\right\} \quad \Longleftrightarrow \quad \begin{array}{c}
\omega_{i}^{A}=f_{i j}^{A}\left(\omega_{j}^{B}, z_{j}^{b}\right), \\
\left.z_{i}^{a}=g_{i j}^{a}\left(\omega_{j}^{B}, z_{j}^{b}\right),\right\}
\end{array}\right\}
$$

with $f_{i j}^{A}\left(0, z_{j}^{b}\right)=0$. Equation (6.1) puts the following constraints on glueing functions:

$$
\begin{aligned}
A_{i j} & =\frac{\partial f_{i j}^{0}}{\partial \omega_{j}^{0}}+\sum_{b} f_{i j}^{b} \frac{\partial g_{i j}^{b}}{\partial \omega_{j}^{0}}+\sum_{\bar{b}} f_{i j}^{\bar{b}} \frac{\partial f_{i j}^{\overline{\bar{b}}}}{\partial \omega_{j}^{0}}, \\
0 & =\frac{\partial f_{i j}^{0}}{\partial \omega_{j}^{a}}+\sum_{b} f_{i j}^{b} \frac{\partial g_{i j}^{b}}{\partial \omega_{j}^{a}}+\sum_{\bar{b}} f_{i j}^{\bar{b}} \frac{\partial f_{i j}^{\overline{\bar{b}}}}{\partial \omega_{j}^{a}}, \\
0 & =\frac{\partial f_{i j}^{0}}{\partial \omega_{j}^{\bar{a}}}+\sum_{b} f_{i j}^{b} \frac{\partial g_{i j}^{b}}{\partial \omega_{j}^{\bar{a}}}+\sum_{\bar{b}} f_{i j}^{\bar{b}} \frac{\partial f_{i j}^{\overline{\bar{b}}}}{\partial \omega_{j}^{\bar{a}}}, \\
A_{i j} \omega_{j}^{\bar{a}} & =\frac{\partial f_{i j}^{0}}{\partial \omega_{j}^{\overline{\bar{a}}}}+\sum_{b} f_{i j}^{b} \frac{\partial g_{i j}^{b}}{\partial \omega_{j}^{\overline{\bar{a}}}}+\sum_{\bar{b}} f_{i j}^{\bar{b}} \frac{\partial f_{i j}^{\overline{\bar{b}}}}{\partial \omega_{j}^{\overline{\bar{a}}}}, \\
A_{i j} \omega_{j}^{a} & =\frac{\partial f_{i j}^{0}}{\partial z_{j}^{a}}+\sum_{b} f_{i j}^{b} \frac{\partial g_{i j}^{b}}{\partial z_{j}^{a}}+\sum_{\bar{b}} f_{i j}^{\bar{b}} \frac{\partial f_{i j}^{\overline{\bar{b}}}}{\partial z_{j}^{a}},
\end{aligned}
$$

which express the fact that the chosen coordinate charts $U_{i}$ are glued by the contactomorphisms.

For any point $t$ in a sufficiently small coordinate neighbourhood $M_{0} \subset M$ of $t_{0}$ with coordinate functions $t^{\alpha}, \alpha=1, \ldots, m=\operatorname{dim} M$, the associated isotropic submanifold $X_{t}=\mu \circ \nu^{-1}(t)$ is given in the domain $U_{i}$ by equations of the form [2]

$$
\omega_{i}^{A}=\phi_{i}^{A}\left(z_{i}^{a}, t^{\alpha}\right), \quad A=0, a, \bar{a}, \overline{\bar{a}}
$$

Lemma 6.1. $X_{t}$ is isotropic if and only if

$$
\phi_{i}^{a}\left(z_{i}, t\right)=-\frac{\partial \phi_{i}^{0}\left(z_{i}, t\right)}{\partial z_{i}^{a}}-\sum_{\bar{b}=p+1}^{n} \phi_{i}^{\bar{b}}\left(z_{i}, t\right) \frac{\partial \phi_{i}^{\overline{\bar{b}}}\left(z_{i}, t\right)}{\partial z_{i}^{a}}
$$

holds. 
Proof. Let $X^{p} \hookrightarrow Y^{2 n+1}$ be an isotropic submanifold in a complex contact manifold $Y$. For an arbitrary $X_{t}$, the deformation of $X$ inside $Y$ is given by

$$
\left.\begin{array}{l}
\omega_{i}^{0}=\phi_{i}^{0}\left(z_{i}, t\right) \\
\omega_{i}^{a}=\phi_{i}^{a}\left(z_{i}, t\right) \\
\omega_{i}^{\bar{a}}=\phi_{i}^{\bar{a}}\left(z_{i}, t\right) \\
\omega_{i}^{\bar{a}}=\phi_{i}^{\bar{a}}\left(z_{i}, t\right)
\end{array}\right\} \Longrightarrow \omega_{i}^{A}=\phi_{i}^{A}\left(z_{i}, t\right)
$$

Then, $\left\{\partial \phi_{i}^{A} /\left.\partial t\right|_{0}\right\}$ is a global section of $N_{X \mid Y} \cdot X_{t}$ is isotropic if and only if

$$
\theta_{i}=\mathrm{d} \omega_{i}^{0}+\omega_{i}^{\bar{a}} \mathrm{~d} \omega_{i}^{\overline{\bar{a}}}+\omega_{i}^{a} \mathrm{~d} z_{i}^{a}
$$

vanishes on $X_{t}$. Then

$$
\begin{aligned}
0 & =\left.\theta_{i}\right|_{X_{t}} \\
& =\mathrm{d} \phi_{i}^{0}\left(z_{i}, t\right)+\phi_{i}^{\bar{a}}\left(z_{i}, t\right) \mathrm{d} \phi_{i}^{\overline{\bar{a}}}\left(z_{i}, t\right)+\phi_{i}^{a}\left(z_{i}, t\right) \mathrm{d} z_{i}^{a} \\
& =\frac{\partial \phi_{i}^{0}\left(z_{i}, t\right)}{\partial z_{i}^{a}} \mathrm{~d} z_{i}^{a}+\phi_{i}^{\bar{a}}\left(z_{i}, t\right) \frac{\partial \phi^{\bar{a}}}{\partial z_{i}^{b}} \mathrm{~d} z_{i}^{b}+\phi_{i}^{a}\left(z_{i}, t\right) \mathrm{d} z_{i}^{a} \\
& =\left[\phi_{i}^{a}\left(z_{i}, t\right)+\frac{\partial \phi_{i}^{0}\left(z_{i}, t\right)}{\partial z_{i}^{a}}+\sum_{\bar{b}=p+1}^{n} \phi_{i}^{\bar{b}}\left(z_{i}, t\right) \frac{\partial \phi_{i}^{\overline{\bar{b}}}\left(z_{i}, t\right)}{\partial z_{i}^{a}}\right] \mathrm{d} z_{i}^{a} .
\end{aligned}
$$

Thus, we obtain

$$
\phi_{i}^{a}\left(z_{i}, t\right)=-\frac{\partial \phi_{i}^{0}\left(z_{i}, t\right)}{\partial z_{i}^{a}}-\sum_{\bar{b}=p+1}^{n} \phi_{i}^{\bar{b}}\left(z_{i}, t\right) \frac{\partial \phi_{i}^{\bar{b}}\left(z_{i}, t\right)}{\partial z_{i}^{a}}
$$

where $\phi_{i}^{A}\left(z_{i}, t\right)$ is a holomorphic function of $z_{i}^{a}$ and $t$, which satisfy the boundary condition $\phi_{i}^{A}\left(z_{i}, t\right)=0$ for $t=t_{0}$.

\section{Isotropic moduli spaces: completeness and maximality}

Let $Y$ be a complex contact manifold and $F \hookrightarrow Y \times M$ be an analytic family of compact complex isotropic submanifolds. The latter is also an analytic family of compact complex submanifolds in the sense of Kodaira and thus, for each $t \in M$, there is a canonical linear map

$$
k_{t}: T_{t} M \rightarrow H^{0}\left(X_{t}, N_{X_{t} \mid Y}\right)
$$

The exact sequence

$$
0 \rightarrow S_{X_{t}} \rightarrow N_{X_{t} \mid Y} \rightarrow J^{1} L_{X_{t}} \rightarrow 0
$$


can be expanded as follows:

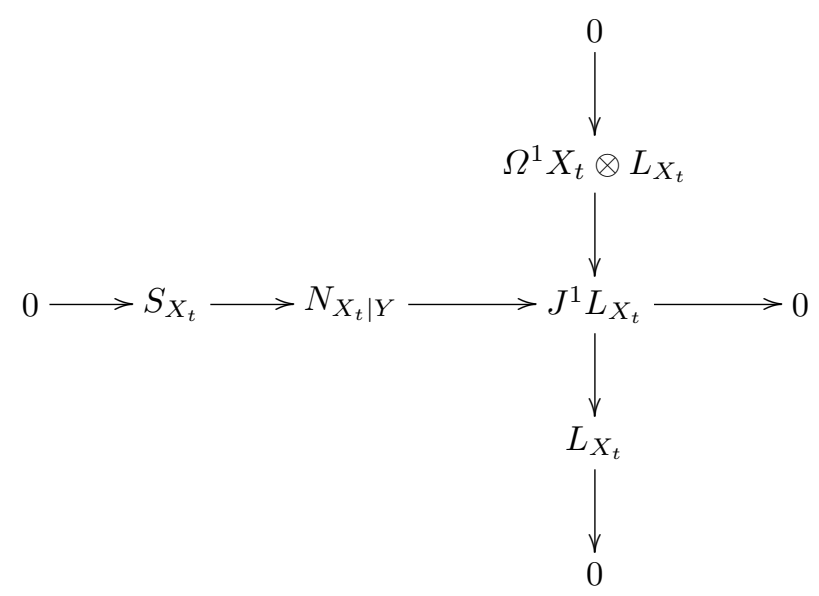

Hence, there is a canonical map represented by a diagonal arrow,

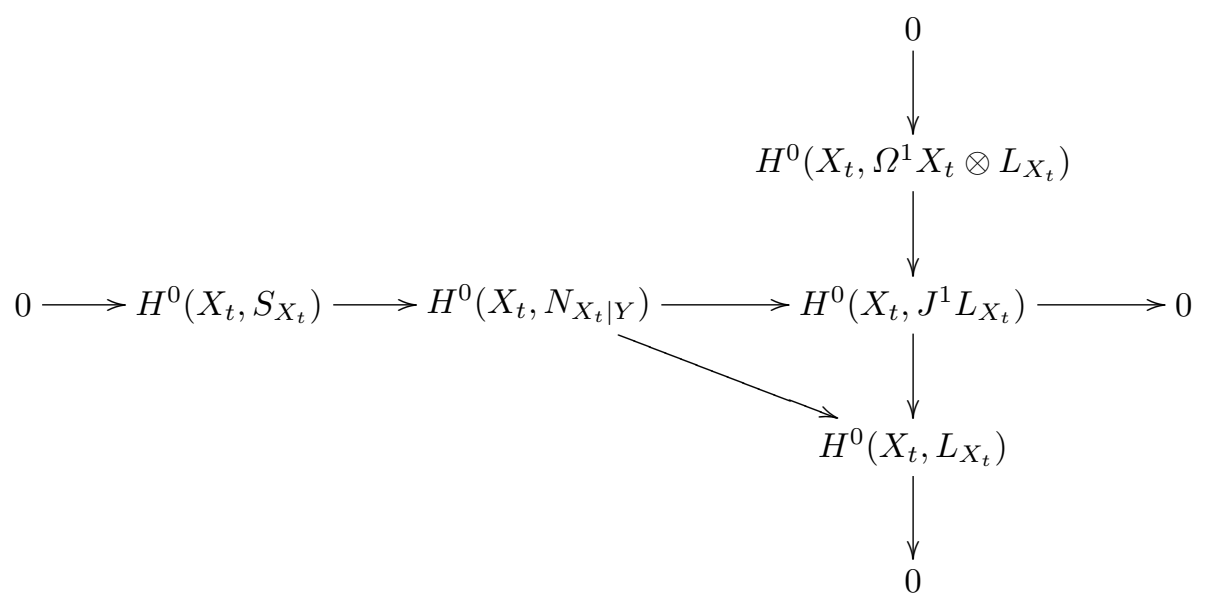

Thus, there is a canonical sequence of linear spaces:

$$
0 \rightarrow H^{0}\left(X_{t}, S_{X_{t}}\right) \rightarrow H^{0}\left(X_{t}, N_{X_{t} \mid Y}\right) \rightarrow H^{0}\left(X_{t}, L_{X_{t}}\right) \rightarrow 0,
$$

which is not exact, in general.

Definition 7.1. The analytic family $F \hookrightarrow Y \times M$ of compact complex isotropic submanifolds is complete at a point $t \in M$ if the Kodaira map $k_{t}$ makes the induced sequence,

$$
0 \rightarrow H^{0}\left(X_{t}, S_{X_{t}}\right) \rightarrow k_{t}\left(T_{t} M\right) \rightarrow H^{0}\left(X_{t}, L_{X_{t}}\right) \rightarrow 0,
$$

exact. The analytic family $F \hookrightarrow Y \times M$ is called complete if it is complete at each point of the moduli space. 
Lemma 7.2 (Ali [1]). If an analytic family $F \hookrightarrow Y \times M$ of compact complex isotropic submanifolds is complete at a point $t_{0} \in M$, then there is an open neighbourhood $U \subseteq M$ of the point $t_{0}$ such that the family $F \hookrightarrow Y \times M$ is complete at all points $t \in U$.

Definition 7.3. An analytic family $F \hookrightarrow Y \times M$ of compact complex isotropic submanifolds is maximal at a point $t_{0} \in M$ if, for any other analytic family $\tilde{F} \hookrightarrow Y \times \tilde{M}$ of compact complex isotropic submanifolds such that $\mu \circ \nu^{-1}\left(t_{0}\right)=\tilde{\mu} \circ \tilde{\nu}^{-1}\left(\tilde{t}_{0}\right)$ for a point $\tilde{t}_{0} \in \tilde{M}$, there exists a neighbourhood $\tilde{U} \subset \tilde{M}$ of $\tilde{t}_{0}$ and a holomorphic map $f: \tilde{U} \rightarrow M$ such that $f\left(\tilde{t}_{0}\right)=t_{0}$ and $\tilde{\mu} \circ \tilde{\nu}^{-1}\left(\tilde{t}^{\prime}\right)=\mu \circ \nu^{-1}\left(f\left(\tilde{t}^{\prime}\right)\right)$ for each $\tilde{t}^{\prime} \in \tilde{U}$. The family $F \hookrightarrow Y \times M$ is called maximal if it is maximal at each point $t$ in the moduli space $M$.

Lemma 7.4 (Ali [1]). If an analytic family of compact complex isotropic submanifolds $F \hookrightarrow Y \times M$ is complete at a point $t_{0} \in M$, then it is maximal at the point $t_{0}$.

\section{Existence theorem}

Theorem 8.1. If $X \hookrightarrow Y$ is a compact complex isotropic submanifold in a complex contact manifold $Y$, then its normal bundle $N_{X \mid Y}$ fits into an extension

$$
0 \rightarrow S_{X} \rightarrow N_{X \mid Y} \rightarrow J^{1} L_{X} \rightarrow 0 .
$$

If $H^{1}\left(X, L_{X}\right)=H^{1}\left(X, S_{X}\right)=0$, then there exists a complete and maximal analytic family $\left\{X_{t} \hookrightarrow Y \mid t \in M\right\}$ of isotropic submanifolds such that

(i) $X_{t_{0}}=X$ for some $t_{0} \in M$;

(ii) the moduli space $M$ is smooth;

(iii) $\operatorname{dim} M=h^{0}\left(X, L_{X}\right)+h^{0}\left(X, S_{X}\right)$;

(iv) the tangent space $T_{t} M, t \in M$, fits into the extension

$$
0 \rightarrow H^{0}\left(X_{t}, S_{X_{t}}\right) \rightarrow k_{t}\left(T_{t} M\right) \rightarrow H^{0}\left(X_{t}, L_{X_{t}}\right) \rightarrow 0 .
$$

Proof. Let $\left(\omega_{i}^{0}, \omega_{i}^{a}, \omega_{i}^{\bar{a}}, \omega_{i}^{\bar{a}}, z_{i}^{a}\right)$ be a coordinate system on $Y$ that is adapted to the isotropic character of the embedding $X \hookrightarrow Y$ as described in $\S 6$. Assume that $\left\{X_{t} \hookrightarrow Y \mid t \in M\right\}$ is a family of compact complex isotropic submanifolds in the complex contact manifold $Y$. According to $\S 6$, such a family can be described by $\phi_{i}^{0}\left(z_{i}, t\right)$, $\phi_{i}^{a}\left(z_{i}, t\right), \phi_{i}^{\bar{a}}\left(z_{i}, t\right), \phi_{i}^{\overline{\bar{a}}}\left(z_{i}, t\right)$, which solve the equations in $U_{i} \cap U_{j}$ :

$$
\begin{aligned}
\phi_{i}^{0}\left(z_{i}, t\right) & =f_{i j}^{0}\left(\phi_{j}^{0}\left(z_{j}, t\right), \phi_{j}^{a}\left(z_{j}, t\right), \phi_{j}^{\bar{a}}\left(z_{j}, t\right), \phi_{j}^{\overline{\bar{a}}}\left(z_{j}, t\right), z_{j}\right), \\
\phi_{i}^{a}\left(z_{i}, t\right) & =f_{i j}^{a}\left(\phi_{j}^{0}\left(z_{j}, t\right), \phi_{j}^{a}\left(z_{j}, t\right), \phi_{j}^{\bar{a}}\left(z_{j}, t\right), \phi_{j}^{\overline{\bar{a}}}\left(z_{j}, t\right), z_{j}\right), \\
\phi_{i}^{\bar{a}}\left(z_{i}, t\right) & =f_{i j}^{\bar{a}}\left(\phi_{j}^{0}\left(z_{j}, t\right), \phi_{j}^{a}\left(z_{j}, t\right), \phi_{j}^{\bar{a}}\left(z_{j}, t\right), \phi_{j}^{\overline{\bar{a}}}\left(z_{j}, t\right), z_{j}\right), \\
\phi_{i}^{\overline{\bar{a}}}\left(z_{i}, t\right) & =f_{i j}^{\overline{\bar{a}}}\left(\phi_{j}^{0}\left(z_{j}, t\right), \phi_{j}^{a}\left(z_{j}, t\right), \phi_{j}^{\bar{a}}\left(z_{j}, t\right), \phi_{j}^{\overline{\bar{a}}}\left(z_{j}, t\right), z_{j}\right), \\
z_{i}^{a} & =g_{i j}^{a}\left(\phi_{j}^{0}\left(z_{j}, t\right), \phi_{j}^{a}\left(z_{j}, t\right), \phi_{j}^{\bar{a}}\left(z_{j}, t\right), \phi_{j}^{\overline{\bar{a}}}\left(z_{j}, t\right), z_{j}\right),
\end{aligned}
$$


and equation (6.8). We know that $N_{X \mid Y}$ fits into a diagram:

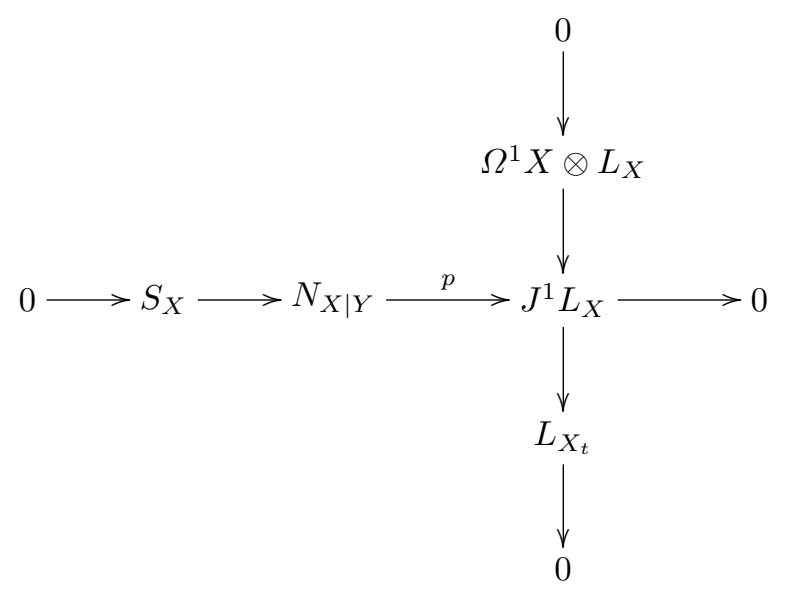

There exists a canonical morphism of sheaves of abelian groups, $\alpha: L_{X} \rightarrow J^{1} L_{X}$, which, in our local coordinates, is given explicitly by

$$
\left\{\phi_{i}^{0}\left(z_{i}, t\right)\right\} \rightarrow\left\{\begin{array}{c}
\phi_{i}^{0}\left(z_{i}, t\right) \\
-\frac{\partial \phi_{i}^{0}\left(z_{i}, t\right)}{\partial z_{i}^{a}}
\end{array}\right\} .
$$

Define a subsheaf of abelian groups in the sheaves $N_{X \mid Y}$ as $\tilde{N}_{X \mid Y}:=p^{-1}\left(\alpha\left(L_{X}\right)\right)$, where $p: N_{X \mid Y} \rightarrow J^{1} L_{X}$ is the canonical epimorphism. By construction, $\tilde{N}_{X \mid Y}$ fits into an exact sequence:

$$
0 \rightarrow S_{X} \rightarrow \tilde{N}_{X \mid Y} \rightarrow L_{X} \rightarrow 0
$$

The long exact sequence associated with the sequence above gives

$$
0 \rightarrow H^{0}\left(X, S_{X}\right) \rightarrow H^{0}\left(X, \tilde{N}_{X \mid Y}\right) \rightarrow H^{0}\left(X, L_{X}\right) \rightarrow H^{1}\left(X, S_{X}\right) \rightarrow \cdots
$$

By assumption, $H^{1}\left(X, S_{X}\right)=0$. Hence, we have an exact sequence of vector spaces,

$$
0 \rightarrow H^{0}\left(X, S_{X}\right) \rightarrow H^{0}\left(X, \tilde{N}_{X \mid Y}\right) \rightarrow H^{0}\left(X, L_{X}\right) \rightarrow 0
$$

implying that

$$
\operatorname{dim} H^{0}\left(X, \tilde{N}_{X \mid Y}\right)=\operatorname{dim} H^{0}\left(X, S_{X}\right)+\operatorname{dim} H^{0}\left(X, L_{X}\right):=m .
$$

Let $\theta_{\alpha}, \alpha=1, \ldots, m$, be a basis of the global sections of $\tilde{N}_{X \mid Y}$. In our coordinate system, each $\theta_{\alpha}$ can be represented by a 0 -cocycle,

$$
\theta_{\alpha} \Longleftrightarrow\left\{\begin{array}{c}
\theta_{\alpha i}^{0} \\
-\frac{\partial \theta_{\alpha i}^{0}}{\partial z_{i}^{a}} \\
\theta_{\alpha i}^{\bar{a}} \\
\theta_{\alpha i}^{\overline{\bar{a}}}
\end{array}\right\}=\left\{\theta_{\alpha i}^{A}\right\}, \quad A=0, a, \bar{a}, \overline{\bar{a}}
$$


In $U_{i} \cap U_{j}$, we have

$$
\theta_{\alpha i}^{A}(z)=F_{i j B}^{A}(z) \theta_{\beta j}^{B}(z), \quad z=\left(0, z_{i}\right),
$$

where the matrix-valued functions are given by

$$
F_{i j B}^{A}=\left[\begin{array}{lccc}
\left.A_{i j}\right|_{X} & 0 & 0 & 0 \\
\left.\frac{\partial f_{i j}^{a}}{\partial \omega_{j}^{0}}\right|_{X} & \left.\frac{\partial f_{i j}^{a}}{\partial \omega_{j}^{b}}\right|_{X} & 0 & 0 \\
\left.\frac{\partial f_{i j}^{\bar{a}}}{\partial \omega_{j}^{0}}\right|_{X} & \left.\frac{\partial f_{i j}^{\bar{a}}}{\partial \omega_{j}^{b}}\right|_{X} & \left.\frac{\partial f_{i j}^{\bar{a}}}{\partial \omega_{j}^{\bar{b}}}\right|_{X} & \left.\frac{\partial f_{i j}^{\bar{a}}}{\partial \omega_{j}^{\overline{\bar{b}}}}\right|_{X} \\
\left.\frac{\partial f_{i j}^{\bar{a}}}{\partial \omega_{j}^{0}}\right|_{X} & \left.\frac{\partial f_{i j}^{\bar{a}}}{\partial \omega_{j}^{b}}\right|_{X} & \left.\frac{\partial f_{i j}^{\overline{\bar{b}}}}{\partial \omega_{j}^{\bar{b}}}\right|_{X} & \left.\frac{\partial f_{i j}^{\overline{\bar{b}}}}{\partial \omega_{j}^{\overline{\bar{b}}}}\right|_{X}
\end{array}\right]
$$

Define

$$
\phi_{i}^{A}\left(z_{i}, t\right)=\left[\begin{array}{c}
\phi_{i}^{0}\left(z_{i}, t\right) \\
\phi_{i}^{a}\left(z_{i}, t\right) \\
\phi_{i}^{\bar{a}}\left(z_{i}, t\right) \\
\phi_{i}^{\bar{a}}\left(z_{i}, t\right)
\end{array}\right],
$$

where equation (6.8) holds. Let $\varepsilon$ be a small positive number. In order to prove theorem 8.1, we must find the holomorphic functions $\phi_{i}^{A}\left(z_{i}, t\right)$ in $z_{i}=\left(z_{i}^{1}, \ldots, z_{i}^{n}\right)$ and in $t=\left(t^{1}, \ldots, t^{m}\right),\left|z_{i}\right|<1,|t|<\varepsilon$, with $\left|\phi_{i}^{A}\left(z_{i}, t\right)\right|<1$ such that

$$
\phi_{i}^{A}\left(g_{i j}^{a}\left(\phi_{j}^{B}\left(z_{j}, t\right), z_{j}\right), t\right)=f_{i j}^{A}\left(\phi_{j}^{B}\left(z_{j}, t\right), z_{j}\right)
$$

where $A=0, a, \bar{a}, \overline{\bar{a}}$, equation (6.8) and the boundary conditions

$$
\phi_{i}^{A}\left(z_{i}, 0\right)=0
$$

and

$$
\left.\frac{\partial \phi_{i}^{A}\left(z_{i}, t\right)}{\partial t^{\alpha}}\right|_{t=0}=\theta_{\alpha i}^{A}(z), \quad z=\left(0, z_{i}\right)
$$

are satisfied. If we succeed in solving all these equations for the functions $\left\{\phi_{i}^{A}\left(z_{i}, t\right)\right\}$, which are holomorphic in $t$ in some neighbourhood $U \subset C^{q}$ of the origin, then the boundary conditions will guarantee that the resulting analytic family $F \hookrightarrow Y \times U$ is complete at $t=0$ and, hence, by Lemmas 7.2 and 7.4, is complete and maximal in some neighbourhood $M \subseteq U$ of the origin. Therefore, all we need to prove the theorem is to solve equations (8.2)-(8.4). We shall do this in three steps.

Step 1 (simplification of the basic system of equations). Let us first show that it is sufficient to solve only those equations of system (8.2), corresponding to $A=0, \bar{a}, \overline{\bar{a}}$, which the holomorphic functions $\left\{\phi_{i}^{A}\left(z_{i}, t\right)\right\}$ satisfy, on overlaps $X \cap U_{i} \cap U_{j}$. Then, denoting

$$
A_{b}^{a}:=\left.\left[\sum_{A=0}^{n} \frac{\partial g_{i j}^{a}}{\partial \omega_{j}^{A}} \frac{\partial \phi_{j}^{A}}{\partial z_{j}^{b}}+\frac{\partial g_{i j}^{a}}{\partial z_{j}^{b}}\right]\right|_{\omega_{j}^{A}=\phi_{j}^{A}\left(z_{j}, t\right)}
$$


and using equations (6.3)-(6.7), we obtain (see [1, pp. 65, 66])

$$
\begin{aligned}
\sum_{a=1}^{n} \frac{\partial \phi_{i}^{0}}{\partial z_{i}^{a}} A_{b}^{a} & =\left.\left[\sum_{a=1}^{n} \frac{\partial \phi_{i}^{0}}{\partial z_{i}^{a}} \sum_{A=0}^{n} \frac{\partial g_{i j}^{a}}{\partial \omega_{j}^{A}} \frac{\partial \phi_{j}^{A}}{\partial z_{j}^{b}}+\frac{\partial g_{i j}^{a}}{\partial z_{j}^{b}}\right]\right|_{\omega_{j}^{A}=\phi_{j}^{A}\left(z_{j}, t\right)} \\
& =\left.\left[\sum_{A=0}^{n} \frac{\partial f_{i j}^{0}}{\partial \omega_{j}^{A}} \frac{\partial \phi_{j}^{A}}{\partial z_{j}^{b}}+\frac{\partial f_{i j}^{0}}{\partial z_{j}^{b}}\right]\right|_{\omega_{j}^{A}=\phi_{j}^{A}\left(z_{j}, t\right)} \\
& =-\sum_{c} f_{i j}^{c} A_{b}^{c}-\sum_{\bar{c}} f_{i j}^{\bar{c}} \frac{\partial f_{i j}^{\bar{c}}}{\partial z_{i}^{a}} A_{b}^{a}
\end{aligned}
$$

which implies that

$$
\sum_{a=1}^{n}\left(\frac{\partial \phi_{i}^{0}}{\partial z_{i}^{a}}+\sum_{\bar{c}} f_{i j}^{\bar{c}} \frac{\partial f_{i j}^{\bar{c}}}{\partial z_{i}^{a}}\right) A_{b}^{a}=-\sum_{c=1}^{n} f_{i j}^{c} A_{b}^{c}
$$

Since the Jacobian of the coordinate transformation

$$
\begin{aligned}
& \left.\operatorname{det} \frac{\partial\left(\omega_{i}^{0}, \omega_{i}^{a}, \omega_{i}^{\bar{a}}, \omega_{i}^{\overline{\bar{a}}}, z_{i}^{a}\right)}{\partial\left(\omega_{j}^{0}, \omega_{j}^{b}, \omega_{j}^{\bar{b}}, \omega_{j}^{\overline{\bar{b}}}, z_{j}^{b}\right)}\right|_{X} \\
& =\left.\left.\left.\left.\left.\frac{\partial f_{i j}^{0}}{\partial \omega_{j}^{0}}\right|_{X} \operatorname{det}\left(\frac{\partial f_{i j}^{a}}{\partial \omega_{j}^{b}}\right)\right|_{X} \operatorname{det}\left(\frac{\partial f_{i j}^{\bar{a}}}{\partial \omega_{j}^{\bar{b}}}\right)\right|_{X} \operatorname{det}\left(\frac{\partial f_{i j}^{\overline{\bar{a}}}}{\partial \omega_{i j}^{\overline{\bar{b}}}}\right)\right|_{X} \operatorname{det}\left(A_{b}^{a}\right)\right|_{t=0}
\end{aligned}
$$

is nowhere zero on $X$, the matrix $A_{b}^{a}$ is non-degenerate at $t=0$ and hence is nondegenerate for all $t$ in some small neighbourhood $U^{\prime}$ of the zero in $C^{m}$. Equation $(8.5)$ then implies that

$$
\left.\left(-\frac{\partial \phi_{i}^{0}}{\partial z_{i}^{a}}-\sum_{\bar{a}} f_{i j}^{\bar{a}} \frac{\partial f_{i j}^{\overline{\bar{a}}}}{\partial z_{i}^{a}}\right)\right|_{z_{i}=g_{i j}\left(\phi_{j}^{B}\left(z_{j}, t\right), z_{j}\right)}=\left.f_{i j}^{a}\right|_{\omega_{j}^{A}=\phi_{j}^{A}\left(z_{j}, t\right)},
$$

i.e. that equation (8.2) with $A=a$ is automatically satisfied. Thus, we must solve equations (8.2) for $A=0, \bar{a}, \overline{\bar{a}}$ with boundary conditions (8.3), (8.4).

Step 2 (existence of formal solutions). In what follows we write the power-series expansion of an arbitrary holomorphic function $P(t)$ in $t^{1}, \ldots, t^{m}$, defined on a neighbourhood of the origin, in the form

$$
P(t)=P_{0}(t)+P_{1}(t)+\cdots+P_{q}(t)+\cdots,
$$

where each term $P_{q}(t)$ denotes a homogeneous polynomial of degree $q$ in $t^{1}, \ldots, t^{m}$, and denote by $P^{[q]}(t)$ the polynomial

$$
P^{[q]}(t)=P_{0}(t)+P_{1}(t)+\cdots+P_{q}(t) .
$$

If $Q(t)$ is another holomorphic function in $t$, we write $P(t) \stackrel{q}{=} Q(t)$ if $P^{[q]}(t)=Q^{[q]}(t)$.

Now we expand each component $\phi_{i}^{A}\left(z_{i}, t\right)$ of $\phi_{i}\left(z_{i}, t\right)$ into a power series

$$
\phi_{i}^{A}\left(z_{i}, t\right)=\phi_{i \mid 1}^{A}\left(z_{i}, t\right)+\cdots+\phi_{i \mid q}^{A}\left(z_{i}, t\right)+\cdots
$$


in $t^{1}, \ldots, t^{m}$, and write

$$
\begin{aligned}
\phi_{i \mid q}^{A}\left(z_{i}, t\right) & =\left(\phi_{i \mid q}^{1}\left(z_{i}, t\right), \ldots, \phi_{i \mid q}^{A}\left(z_{i}, t\right), \ldots, \phi_{i \mid q}^{p}\left(z_{i}, t\right)\right), \\
\phi_{i}^{A[q]}\left(z_{i}, t\right) & =\phi_{i \mid 1}^{A}\left(z_{i}, t\right)+\cdots+\phi_{i \mid q}^{A}\left(z_{i}, t\right) .
\end{aligned}
$$

The equality (8.2) is then reduced to the following system of congruences:

$$
\phi_{i}^{A[q]}\left(g_{i j}^{a}\left(\phi_{j}^{B[q]}\left(z_{j}, t\right), z_{j}\right), t\right) \stackrel{q}{=} f_{i j}^{A}\left(\phi_{j}^{B[q]}\left(z_{j}, t\right), z_{j}\right), \quad q=1,2,3, \ldots
$$

We note that the congruence $(8.6)_{1}$ is equivalent to

$$
\phi_{i \mid 1}^{A}\left(z_{i}, t\right)=F_{i j B}^{A}(z) \cdot \phi_{j \mid 1}^{B}\left(z_{j}, t\right), \quad z=\left(0, z_{i}\right)=\left(0, z_{j}\right) .
$$

First, we shall construct the polynomials $\phi_{i}^{A[q]}\left(z_{i}, t\right)$ by induction on $q$. In view of the boundary conditions (8.3), (8.4), we define

$$
\phi_{i \mid 1}^{A}\left(z_{i}, t\right)=\sum_{\alpha} \theta_{\alpha i}^{A}(z) t^{\alpha} .
$$

It is clear by (8.1) that the linear forms $\phi_{i \mid 1}^{A}\left(z_{i}, t\right), i \in I$, satisfy $(8.6)_{1}$.

Assume that the polynomials $\phi_{i}^{A[q]}\left(z_{i}, t\right), i \in I$, satisfying $(8.6)_{q}$ are already determined for an integer $q \geqslant 1$. For the sake of simplicity we write

$$
\begin{aligned}
\phi_{j}^{A[q]}(t) & =\phi_{j}^{A[q]}\left(z_{j}, t\right), \\
f_{i j}^{A}\left(\omega_{j}^{B}\right) & =f_{i j}^{A}\left(\omega_{j}^{B}, z_{j}\right), \\
f_{k j}^{A}\left(\omega_{j}^{B}\right) & =f_{k j}^{A}\left(\omega_{j}^{B}, z_{j}\right), \\
g_{i j}^{a}\left(\omega_{j}^{B}\right) & =g_{i j}^{a}\left(\omega_{j}^{B}, z_{j}\right),
\end{aligned}
$$

and we set

$$
\left.\psi_{i j}^{A}\left(z_{j}, t\right) \stackrel{q+1}{=} \phi_{i}^{A[q]}\left(z_{i}, t\right)\right|_{z_{i}^{a}=g_{i j}^{a}\left(\phi_{j}^{B[q]}\left(z_{j}, t\right), z_{j}\right)}-\left.f_{i j}^{A}\left(\omega_{j}^{B}, z_{j}\right)\right|_{\omega_{j}^{B}=\phi_{j}^{B[q]}\left(z_{j}, t\right)} .
$$

Note that $\psi_{i j}^{A}\left(z_{j}, t\right)$ is a homogeneous polynomial of degree $q+1$ in $t^{1}, \ldots, t^{m}$ whose coefficients are vector-valued holomorphic functions of $z_{j},\left|z_{j}\right|<1,\left|g_{i j}\left(0, z_{j}\right)\right|<1$, and that

$$
\psi_{i j}^{A}\left(z_{j}, t\right) \stackrel{q+1}{\equiv} \phi_{i}^{A[q]}\left(g_{i j}\left(\phi_{j}^{B[q]}(t)\right), t\right)-f_{i j}^{A}\left(\phi_{j}^{B[q]}(t)\right) .
$$

We define

$$
\psi_{i j}^{A}(z, t)=\psi_{i j}^{A}\left(z_{j}, t\right) \quad \text { for } z=\left(0, z_{j}\right) \in U_{i} \cap U_{j} .
$$

We have the equality $[\mathbf{1}]$

$$
\psi_{i j}^{A}(z, t)=\psi_{i k}^{A}(z, t)+F_{i k B}^{A}(z) \cdot \psi_{k j}^{B}(z, t) \quad \text { for } z \in U_{i} \cap U_{j} \cap U_{k} .
$$


We now have to prove that the 1-cocycle $\left\{\psi_{i j}^{A}\left(z_{i}, t\right)\right\}$ takes values in $\tilde{N}_{X \mid Y}$ rather than in $N_{X \mid Y}$. By definition, we obtain

$$
\left.\psi_{i j}^{0}\left(z_{j}, t\right) \stackrel{q+1}{=} \phi_{i}^{0[q]}\left(z_{i}, t\right)\right|_{z_{i}^{a}=g_{i j}^{a}\left(\phi_{j}^{B[q]}\left(z_{j}, t\right), z_{j}\right)}-\left.f_{i j}^{0}\left(\omega_{j}^{B}, z_{j}\right)\right|_{\omega_{j}^{B}=\phi_{j}^{B[q]}\left(z_{j}, t\right)}
$$

and

$$
\left.\psi_{i j}^{a}\left(z_{j}, t\right) \stackrel{q+1}{=} \phi_{i}^{a[q]}\left(z_{i}, t\right)\right|_{z_{i}^{a}=g_{i j}^{a}\left(\phi_{j}^{B[q]}\left(z_{j}, t\right), z_{j}\right)}-\left.f_{i j}^{a}\left(\omega_{j}^{B}, z_{j}\right)\right|_{\omega_{j}^{B}=\phi_{j}^{B[q]}\left(z_{j}, t\right)} .
$$

Then $\left\{\psi_{i j}^{A}\left(z_{j}, t\right)\right\}$ represents a cohomology class in $H^{1}\left(X, \tilde{N}_{X \mid Y}\right)$ if and only if

$$
\psi_{i j}^{a}\left(z_{j}, t\right)=-\frac{\partial \psi_{i j}^{0}\left(z_{j}, t\right)}{\partial z_{j}^{b}}\left(A^{-1}\right)_{a}^{b}
$$

or

$$
\frac{\partial \psi_{i j}^{0}\left(z_{j}, t\right)}{\partial z_{j}^{b}}=-\sum_{a} \psi_{i j}^{a}\left(z_{j}, t\right) A_{b}^{a} .
$$

To prove this, differentiate (8.10) with respect to $z_{j}^{b}$, and using equations (6.3)-(6.7) and (8.11) with Lemma 6.1 , we obtain (see [1] )

$$
\begin{aligned}
\frac{\partial \psi_{i j}^{0}}{\partial z_{j}^{b}}= & \left.\frac{\partial \phi_{i}^{0[q]}\left(z_{i}, t\right)}{\partial z_{i}^{a}}\right|_{z_{i}^{a}=g_{i j}^{a}\left(\phi_{j}^{B[q]}\left(z_{j}, t\right), z_{j}\right)}\left(\frac{\partial g_{i j}^{a}}{\partial \omega_{j}^{A}} \frac{\partial \phi_{j}^{A}}{\partial z_{j}^{b}}+\frac{\partial g_{i j}^{a}}{\partial z_{j}^{b}}\right) \\
& -\left.\frac{\partial f_{i j}^{0}}{\partial \omega_{j}^{B}}\right|_{\omega_{j}^{B}=\phi_{j}^{B[q]}\left(z_{j}, t\right)} \frac{\partial \phi_{j}^{B[q]}}{\partial z_{j}^{b}}-\left.\frac{\partial f_{i j}^{0}}{\partial z_{j}^{b}}\right|_{\omega_{j}^{B}=\phi_{j}^{B[q]}\left(z_{j}, t\right)} \\
= & -\phi_{i}^{a[q]} A_{b}^{a}-\sum_{\bar{b}} \phi_{i}^{\bar{b}[q]} \frac{\partial \phi_{i}^{\overline{\bar{b}}[q]}}{\partial z_{i}^{a}} A_{b}^{a}+\left.\sum_{a} f_{i j}^{a}\right|_{\omega_{j}^{B}=\phi_{j}^{B[q]}\left(z_{j}, t\right)} A_{b}^{a}+\sum_{\bar{b}} \phi_{i}^{\bar{b}[q]} \frac{\partial \phi_{i}^{\bar{b}}[q]}{\partial z_{i}^{a}} A_{b}^{a} \\
= & -\sum_{a} \psi_{i j}^{a}\left(z_{j}, t\right) A_{b}^{a} .
\end{aligned}
$$

Hence,

$$
\frac{\partial \psi_{i j}^{0}\left(z_{j}, t\right)}{\partial z_{j}^{b}}=-\sum_{a} \psi_{i j}^{a}\left(z_{j}, t\right) A_{b}^{a} .
$$

From the exact sequence

$$
0 \rightarrow S_{X} \rightarrow \tilde{N}_{X \mid Y} \rightarrow L_{X} \rightarrow 0
$$

it follows that

$$
\cdots \rightarrow H^{1}\left(X, S_{X}\right) \rightarrow H^{1}\left(X, \tilde{N}_{X \mid Y}\right) \rightarrow H^{1}\left(X, L_{X}\right) \rightarrow \cdots
$$

as $H^{1}\left(X, S_{X}\right)=H^{1}\left(X, L_{X}\right)=0$, and hence we get $H^{1}\left(X, \tilde{N}_{X \mid Y}\right)=0$. Therefore, there exists a collection $\left\{\phi_{i \mid q+1}^{A}(z, t)\right\}$ of homogeneous polynomials $\phi_{i \mid q+1}^{A}(z, t)$ of degree $q+1$ in $t^{1}, \ldots, t^{m}$, whose coefficients are holomorphic functions of $z$ defined on $U_{i}$ if we take values in $\tilde{N}_{X \mid Y}$ such that

$$
\psi_{i j}^{A}(z, t)=F_{i j B}^{A}(z) \phi_{j \mid q+1}^{B}(z, t)-\phi_{i \mid q+1}^{A}(z, t) \quad \text { for } z \in U_{i} \cap U_{j} .
$$


Considering the coefficients of $\phi_{i \mid q+1}^{A}(z, t)$ as functions of the local coordinate $z_{i}$ of $z$, we write $\phi_{i \mid q+1}^{A}\left(z_{i}, t\right)$ for $\phi_{i \mid q+1}^{A}(z, t)$. The formula (8.12) can then be written in the form

$$
\psi_{i j}^{A}\left(z_{j}, t\right)=F_{i j B}^{A}(z) \phi_{j \mid q+1}^{B}\left(z_{j}, t\right)-\phi_{i \mid q+1}^{A}\left(g_{i j}\left(0, z_{j}\right), t\right) .
$$

We now define

$$
\phi_{i}^{A[q+1]}\left(z_{i}, t\right)=\phi_{i}^{A[q]}\left(z_{i}, t\right)+\phi_{i \mid q+1}^{A}\left(z_{i}, t\right), \quad i \in I .
$$

On writing $\phi_{j}^{A[q+1]}(t)$ for $\phi_{j}^{A[q]}\left(z_{j}, t\right)$, we then have

$$
\begin{gathered}
\phi_{i}^{A[q+1]}\left(g_{i j}\left(\phi_{j}^{B[q+1]}(t)\right), t\right) \stackrel{q+1}{\equiv} \phi_{i}^{A[q]}\left(g_{i j}\left(\phi_{j}^{B[q]}(t)\right), t\right)+\phi_{i \mid q+1}^{A}\left(g_{i j}\left(0, z_{j}\right), t\right), \\
f_{i j}^{A}\left(\phi_{j}^{B[q+1]}(t)\right) \stackrel{q+1}{\equiv} f_{i j}^{A}\left(\phi_{j}^{B[q]}(t)\right)+F_{i j B}^{A}(z) \phi_{j \mid q+1}^{B}\left(z_{j}, t\right) .
\end{gathered}
$$

Consequently, from (8.8) and (8.9), we obtain the congruence

$$
\phi_{i}^{A[q+1]}\left(g_{i j}\left(\phi_{j}^{B[q+1]}(t)\right), t\right) \stackrel{q+1}{\equiv} f_{i j}^{A}\left(\phi_{j}^{B[q+1]}(t)\right) .
$$

This completes our inductive construction of the polynomials $\phi_{i}^{A[q]}\left(z_{i}, t\right), i \in I$, satisfying $(8.6)_{q}$. Thus, setting

$$
\phi_{i}^{A}\left(z_{i}, t\right)=\phi_{i \mid 1}^{A}\left(z_{i}, t\right)+\cdots+\phi_{i \mid q}^{A}\left(z_{i}, t\right)+\cdots,
$$

we obtain a formal power series $\phi_{i}^{A}\left(z_{i}, t\right), i \in I$, in $t^{1}, \ldots, t^{m}$, whose coefficients are vector-valued holomorphic functions of $z_{i},\left|z_{i}\right|<1$, which satisfies equations (8.2)-(8.4).

Step 3 (convergence). There is an arbitrariness involved in the construction of the formal power series $\phi_{i}^{A}\left(z_{i}, t\right)$. For each $q \geqslant 1$, the 0 -cochain $\left\{\phi_{i \mid q+1}^{A}\left(z_{i}, t\right)\right\}$, whose image under the coboundary map is the 1-cocycle $\left\{\psi_{i j}^{A}\left(z_{j}, t\right)\right\}$, is defined up to the addition of a global holomorphic section of $\tilde{N}_{X \mid Y}$ over $X$. We now want to use this freedom to ensure convergence of the formal constructions. The idea is to estimate each holomorphic function involved in the construction of $\phi_{i}^{A}\left(z_{i}, t\right)$ and show that, under appropriate choices of $\left\{\phi_{i \mid q+1}^{A}\left(z_{i}, t\right)\right\}, q=1,2, \ldots$, all the resulting power series $\left\{\phi_{i}^{A}\left(z_{i}, t\right)\right\}$ are majorities by an obviously convergent series

$$
A(t)=\frac{a}{16 b} \sum_{n=1}^{\infty} \frac{b^{n}}{n^{2}}\left(t_{1}+t_{2}+\cdots+t_{m}\right)^{n},
$$

where $a$ and $b$ are some positive constants. Fortunately, what really counts at this stage is the compactness of $X$ and the analyticity of all functions involved in the construction. Therefore, all the estimates obtained by Kodaira [4] carry over verbatim to our case. We conclude that polynomials $\phi_{i \mid q+1}^{A}\left(z_{i}, t\right)$ can be chosen in such a way that the power series $\phi_{i}^{A}\left(z_{i}, t\right)$ converges for $|t|<\varepsilon$, where $\varepsilon$ is some positive number. This completes the proof of Theorem 8.1. 
Example 8.2. Let $Y$ be a five-dimensional complex projective space $\mathcal{C} \mathcal{P}^{5}$ with contact structure coming from some non-degenerate skew symmetric product $\omega$ on $\mathcal{C}^{6}$. The contact line bundle $L$ of such a structure is $\mathcal{O}(2)$. Let $X=\mathcal{C P}^{1}$ be an isotropic complex projective line in $Y$ such that $L_{X}=\mathcal{O}_{X}(2)$. The normal bundle of $X \hookrightarrow Y$ is $N_{X \mid Y}=\mathcal{C}^{4} \otimes \mathcal{O}_{X}(1)$. Since $J^{1} L_{X}=\mathcal{C}^{2} \otimes \mathcal{O}_{X}(1)$, the exact sequence,

$$
0 \rightarrow S_{X} \rightarrow N_{X \mid Y} \rightarrow J^{1} L_{X} \rightarrow 0,
$$

implies that $S_{X} \simeq \mathcal{C}^{2} \otimes \mathcal{O}_{X}(1)$. As $H^{1}\left(X, L_{X}\right)=H^{1}\left(X, S_{X}\right)=0$, Theorem 8.1 then ensures that there is a $(3+4)=7$-dimensional moduli space $M$ of deformations of $X$ in the class of isotropic submanifolds.

In fact, $X$ is a complex projective line, linearly embedded in $\mathcal{C} \mathcal{P}^{5}$ in the usual way. Nonprojectively, this corresponds to a 2-plane in $\mathcal{C}^{6}$, and the condition that $\mathcal{C} \mathcal{P}^{1}$ is isotropic with respect to the contact structure translates into the condition that the 2-plane is isotropic with respect to the symplectic form $\omega$.

Let us consider first the linear deformations of $X$. These correspond to a subset of the Grassmannian of all 2-planes in $\mathcal{C}^{6}$ which has dimension $2(6-2)=8$. We may embed this Grassmannian in $\mathcal{P}\left(\wedge^{2} \mathcal{C}^{6}\right)=\mathcal{C} \mathcal{P}^{14}$ by the Plücker embedding. The isotropic 2-planes then correspond to a hyperplane section of the image of this Grassmannian, since the symplectic form $\omega$ is a linear functional on $\wedge^{2} \mathcal{C}^{6}$. The space of isotropic 2-planes therefore has complex dimension 7 . Therefore, we can identify the moduli space $M$ of deformations of $X$ with the isotropic Grassmannian of 2-planes in $\mathcal{C}^{6}$.

\section{References}

1. M. S. ALI, Moduli spaces of compact complex isotropic submanifolds in complex contact manifold, PhD thesis, Department of Mathematics, University of Glasgow, UK (2003).

2. V. I. ARNol'D, Mathematical methods of classical mechanics (Springer, 1978).

3. K. Kodaira, A theorem of completeness of characteristic systems for analytic families of compact submanifolds of complex manifolds, Ann. Math. 75 (1962), 146-162.

4. K. Kodaira, Complex manifolds and deformation of complex structures (Springer, 1986).

5. C. LeBrun, Thickenings and conformal gravity, Commun. Math. Phys. 139 (1991), 1-43.

6. S. A. Merkulov, Geometry of Kodaira moduli spaces, Proc. Am. Math. Soc. 124 (1996), 1499-1506.

7. S. A. Merkulov, Existence and geometry of Legendre moduli spaces, Math. Z. 226 (1997), 211-265.

8. S. A. Merkulov, Affine connections on involutive G-structures, in Geometry and physics, Lecture Notes in Pure and Applied Mathematics, Volume 184, pp. 261-274 (Dekker, New York, 1997). 